\title{
Experience with Tetralogy of Fallot Patients in a Tertiary Care Teaching Institute of North India
}

${ }^{1}$ Rajarajan Ganesan, ${ }^{2}$ Subhrashis G Niyogi, ${ }^{3}$ Goverdhan D Puri, ${ }^{4}$ Avneet Singh, ${ }^{5}$ Krishna P Gourav, ${ }^{6}$ Rupesh Kumar, ${ }^{7}$ Indranil Biswas, ${ }^{8}$ Mandeep Kumar, ${ }^{9}$ Imran Bhat, ${ }^{10}$ Banashree Mandal

\section{ABSTRACT}

Tetralogy of fallot (TOF) is the most common cyanotic congenital heart disease. Anatomical variations occurring in these patients often contribute to postoperative morbidity and affect the long term outcome. However, there is limited literature discussing the perioperative imaging and management of these variations. In this review, we highlight the common anatomical variations we encountered at our institute during routine perioperative echocardiography. Discussion of these with the operating team helped us in improving the surgical outcome.

Keywords: Echocardiography, Innominate vein, Tetralogy of fallot

How to cite this article: Ganesan R, Niyogi SG, Puri GD, Singh A, Gourav KP, Kumar R, Biswas I, Kumar M, Bhat I, Mandal B. Experience with Tetralogy of Fallot Patients in a Tertiary Care Teaching Institute of North India. J Perioper Echocardiogr 2018;6(2):51-56.

\section{Source of support: Nil}

Conflict of interest: None

\section{OUR EXPERIENCE WITH TETRALOGY OF FALLOT PATIENTS}

Tetralogy of Fallot (TOF) is the most common cyanotic congenital heart disease, and it accounts for $23 \%$ of the pediatric cardiac surgical cases operated at our institute. Preoperative transthoracic and pre-bypass transesophageal echocardiography complement each other in defining the finer details of anatomy, and therefore, guide surgical management. The basic transesophageal echocardiography in TOF patients has been discussed in

\footnotetext{
${ }^{1}$ Senior Resident, ${ }^{2,4,5,7,9}$ Fellow, ${ }^{3}$ Professor and Head, ${ }^{6}$ Assistant Professor, ${ }^{8}$ Junior Resident, ${ }^{10}$ Additional Professor

1-5,7-10Department of Anesthesia and Intensive Care, Postgraduate Institute of Medical Education and Research, Chandigarh, India

${ }^{6}$ Department of Cardiovascular and Thoracic Surgery, Postgraduate Institute of Medical Education and Research, Chandigarh, India

Corresponding Author: Banashree Mandal, Additional Professor, Department of Anesthesia and Intensive Care, Postgraduate Institute of Medical Education and Research, Chandigarh, India, e-mail: banashreemandal@yahoo.co.in
}

a previous review. ${ }^{1}$ Here, we discuss some of the common variations of TOF that we have encountered at our institute.

Placing the transthoracic echo probe in the precordial or subcostal window to obtain the short axis view of the heart visualizes the right ventricle outflow tract (RVOT) and the ventricular septal defect (VSD). While placing the continuous wave Doppler on RVOT, the angle between the Doppler and blood flow should be less than 20 degrees to ensure an error of less than $10 \%{ }^{2}$ Another important feature to note is the location of the VSD. The common location of VSD in TOF is perimembranous/subaortic, which can be visualized adjacent to the septal tricuspid leaflet and the aortic valve in short axis view. On the other hand, the VSD is subarterial in around $15 \%$ of TOF patients, presenting for repair, in India. ${ }^{3} \mathrm{~A}$ subarterial VSD is identified on echo by its location more anteriorly, away from the tricuspid valve and adjacent to the pulmonary annulus (Fig. 1 and Video 1). Because of its location adjacent to the annuli of both great vessels, suturing the upper margin of the VSD patch requires care. Its other surgical implication is the more frequent requirement of a transannular patch. ${ }^{3}$

Another possible variation in the VSD in a TOF patient is the presence of a restrictive VSD. A perimembranous VSD in TOF can rarely (around $1.5 \%$ incidence) ${ }^{4}$ be partially closed by the septal tricuspid leaflet (Fig. 2 and Video 2). This can adversely affect the outcome because of the supra systemic right ventricle pressure

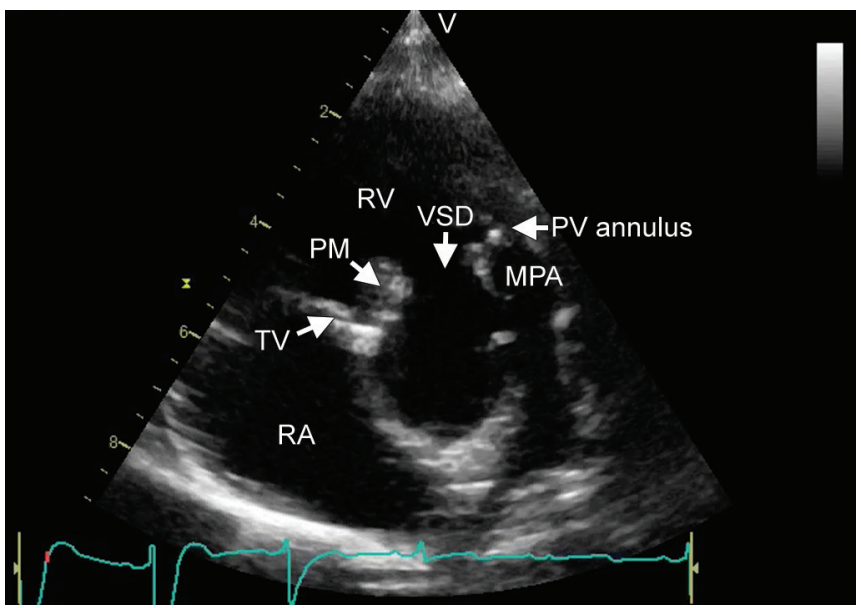

Fig. 1: Parasternal right ventricle inflow outflow view in a tetralogy of Fallot patient with sub-arterial ventricular septal defect. Note the sub-arterial ventricular septal defect, which is away from the tricuspid valve and located more anteriorly, adjacent to pulmonary annulus 


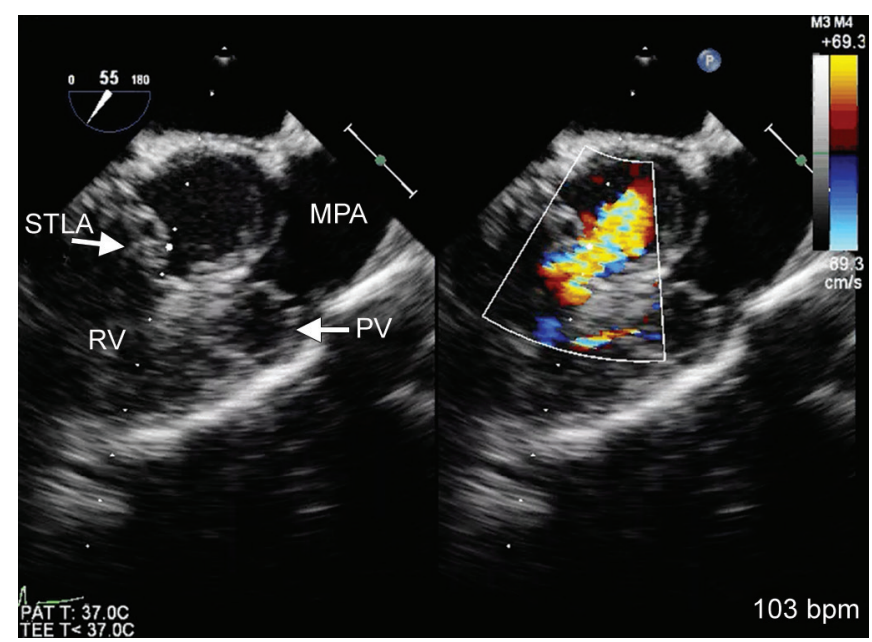

Fig. 2: Midesophageal right ventricle inflow outflow view in a tetralogy of Fallot patient with restrictive ventricular septal defect. Note the ventricular septal defect being closed by the septal tricuspid leaflet and a doming pulmonary valve causing severe right ventricular hypertrophy

and severe right ventricle hypertrophy. Usually, the large VSD in a classic TOF patient provides a low-pressure outflow for the right ventricle (RV). Any restriction in VSD size increases the RV pressure and, thereby, can lead to excessive RV hypertrophy and early RV failure. Adequate RV muscle bundle resection for achieving a low-pressure ratio of right ventricle/left ventricle can also be challenging in such cases. A small interatrial or interventricular communication may be deliberately created in such cases for decompressing the right ventricle postoperatively.

We follow up the characterization of the VSD with an examination of the pulmonary valve. The more anterior pulmonary valve may be better visualized by transthoracic echocardiography. Nevertheless, the upper oesophageal aortic arch short axis view can be an alternative view when the valve is not clearly visualized in the mid esophageal right ventricle inflow-outflow view. This upper esophageal view (Fig. 3 and Video 3) also provides good alignment of doppler wave for measuring gradient across the pulmonary valve. When the pulmonary valve cusps demonstrate doming (Fig. 2 and Video 2), i.e., the valve cusps move away from each other at the base, but are restricted at the tips of the cusps, it can indicate the presence of bicuspid pulmonary valve or commissural fusion in a tricuspid pulmonary valve. The feasibility of doing a pulmonary valve-sparing surgery in the form of commissurotomy and repair is determined by the presence of a bicuspid or tricuspid valve, along with measurement of annulus size and the presence of dysplasia. This gains significance because the goal of intracardiac repair for tetralogy of Fallot is to abolish cyanosis from shunting and reduce right ventricle pressure overload. Selected cases may benefit

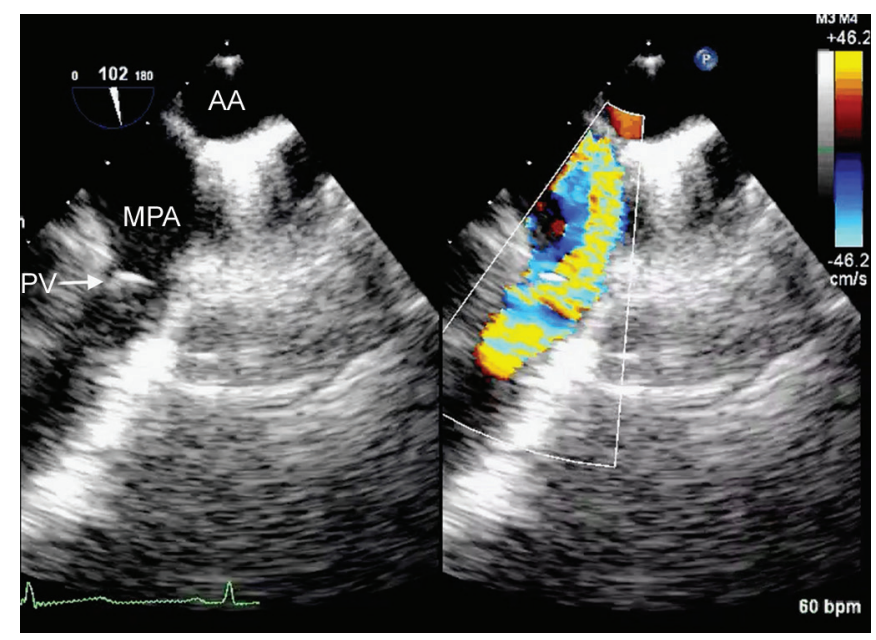

Fig. 3: Upper oesophageal aortic arch short axis view in a tetralogy of Fallot patient with pulmonary valvular stenosis. Note the ability to visualize the doming pulmonary valve in the near field of echo and the good orientation of flow for doppler placement

from a pulmonary valve-sparing approach, where no transannular patch is placed, thus reducing the severity of pulmonary regurgitation and possibly resulting in reduced long term morbidity. ${ }^{5}$ Another option to reduce pulmonary regurgitation is the creation of a neo-pulmonary valve. (Figs 4 and 5 and Videos 4 and 5) show such a monocuspid neo-pulmonary valve prepared from the pericardium.

We next measure the dimensions of the main pulmonary artery and branch pulmonary arteries. General anesthesia with controlled ventilation offers an advantage in measuring the diameter of the left pulmonary artery by transthoracic echocardiography in the left upper parasternal view by providing an opportunity for apnea (Fig. 6 and Video 6). The continuous flow from a patent ductus arteriosus or a Blalock-Tausig shunt can also be seen in this view. In patients with a large PDA, continuous flow can be seen in the pulmonary artery in midesophageal view, especially in the presence of severe RVOT obstruction causing minimal antegrade flow (Fig. 7 and Video 7).

The right pulmonary artery diameter can be measured in the near field in the mid-esophageal ascending aorta long axis view. One anomaly which can mimic the right pulmonary artery in this view is the retroaortic innominate vein (Fig. 8 and Video 8). ${ }^{6}$ Normally, innominate vein passes above the aortic arch and joins the superior vena cava. In $8.5 \%$ of patients with $\mathrm{TOF}^{7}$ an innominate vein passing below the aortic arch has been reported. This can be identified on echo by the venous flow pattern on doppler. When this is present, there is a possible difficulty in left-sided central line placement and the need for a more caudal surgical superior vena cava cannulation to avoid venous obstruction during cardiopulmonary bypass. ${ }^{8}$ It's differential diagnosis includes, among others, major aorta pulmonary collaterals. 


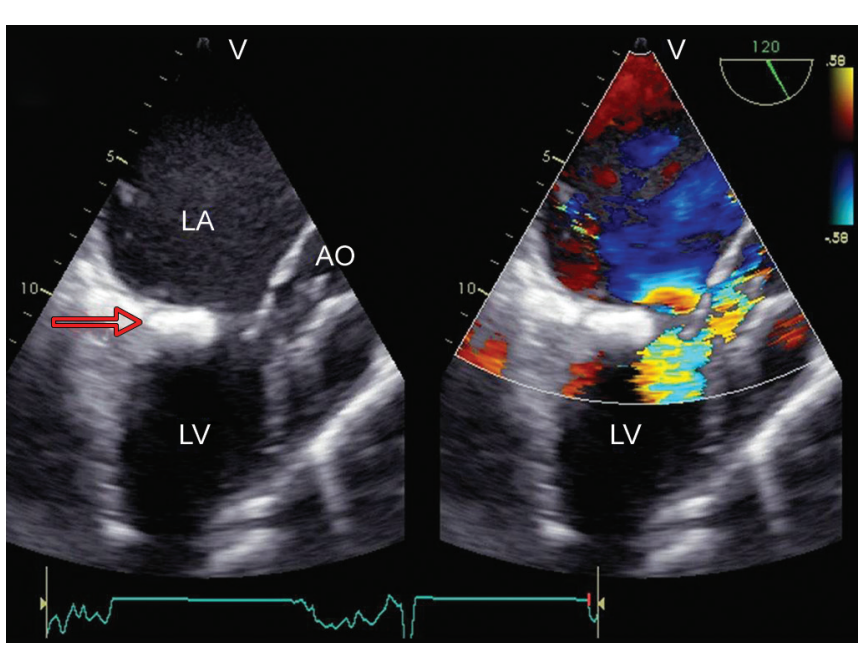

Fig. 4: Transgastric right ventricle outflow view in a patient with repaired tetralogy of Fallot with a monocuspid neo-pulmonary valve. PV, Neo-pulmonary valve; RV, Right ventricle.

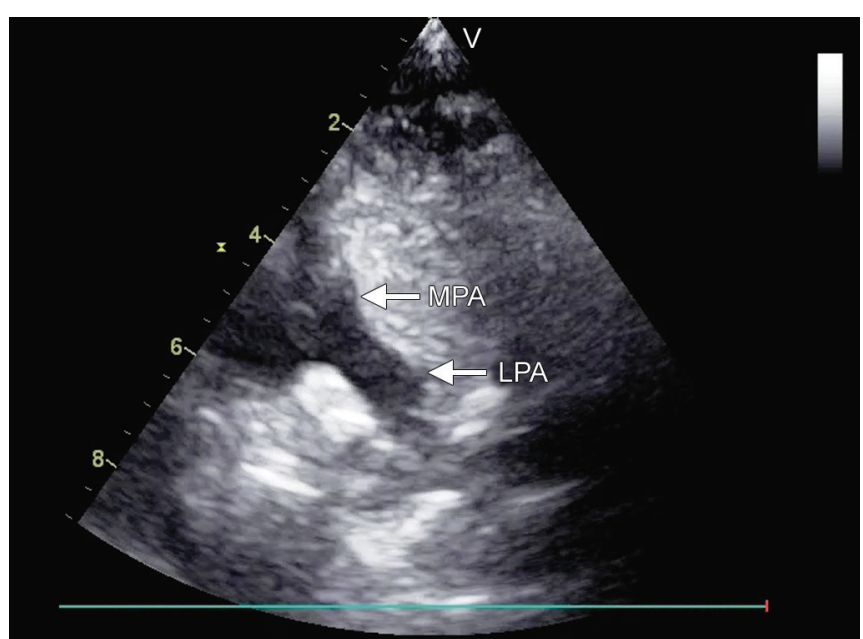

Fig. 6: Upper left parasternal view during apnea showing the pulmonary artery bifurcation and a good view of the left pulmonary artery. LPA, Left pulmonary artery; MPA, Main pulmonary artery

The other venous anomaly which needs to be identified before cannulation is the persistent left superior vena cava. It often drains into the coronary sinus and its presence is suspected by the presence of a dilated coronary sinus (Fig. 9), or the failure to locate the innominate vein after sternotomy. Confirmation of its presence is by microbubble injection through a left-sided venous cannula. When present, the surgeon may choose to place a cannula in the coronary sinus (Fig. 9 and Video 9) or directly in the left-sided superior vena cava and connect it to pump suction for achieving venous drainage. ${ }^{9}$ TEE confirmation of the position of the catheter avoids flooding of the field with blood after atriotomy. In the presence of the central venous catheter in the right internal jugular vein, visual inspection of the face remains the only sign left for monitoring increase in upper body left-sided venous pressure.

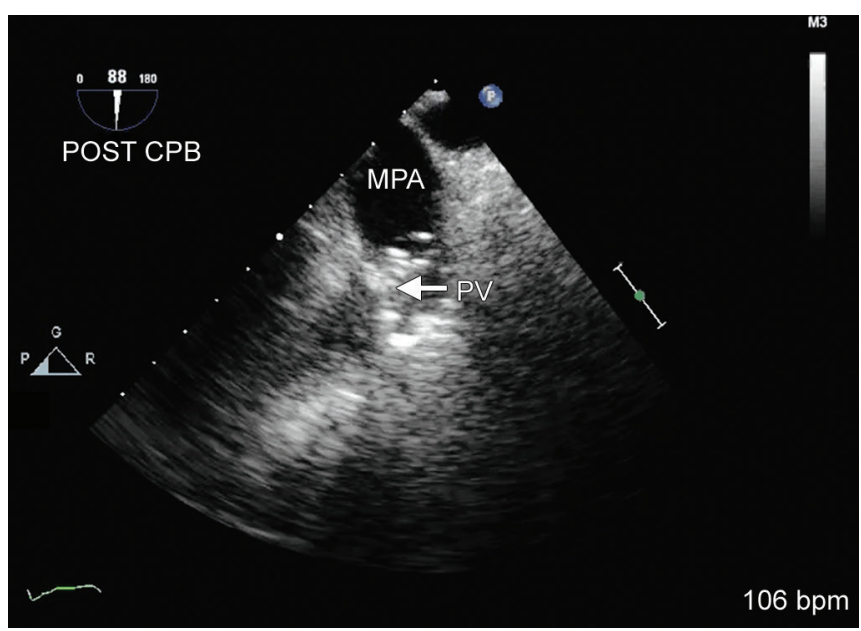

Fig. 5: Upper oesophageal aortic arch short axis view in a patient with repaired tetralogy of Fallot with monocuspid neo-pulmonary valve. MPA, Main pulmonary artery; PV, Neo-pulmonary valve

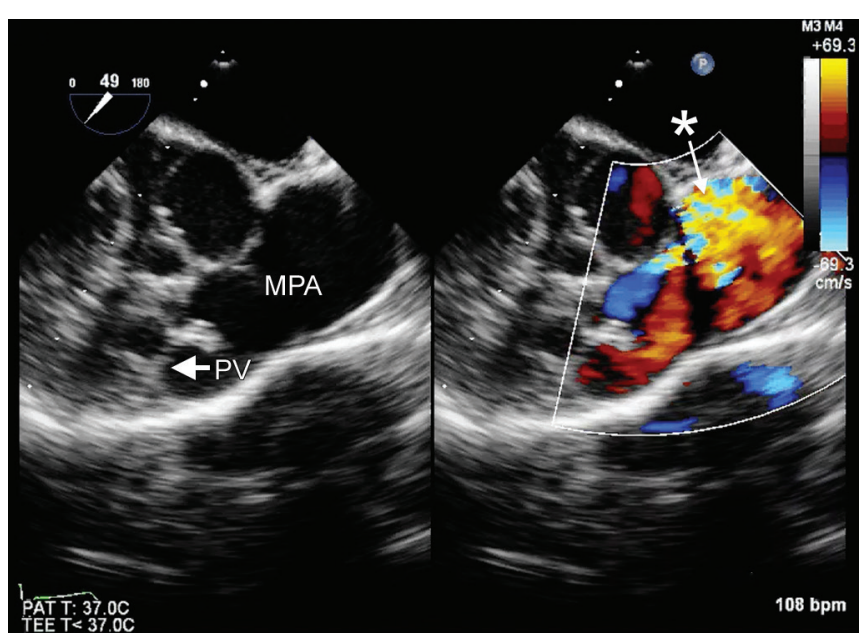

Fig. 7: Mid-esophageal view in a tetralogy of Fallot patient demonstrating the blood flow from a large patent ductus arteriosus into the main pulmonary artery.*- Blood flow into the main pulmonary artery from the patent ductus arteriosus.

The commonly seen vascular anomaly in TOF is the presence of a right-sided aortic arch (13-34\%). ${ }^{10}$ The rightsided arch in TOF usually has a mirror image branching pattern of normal. The descending aorta lies on the right side of the vertebral column and is visualized by turning the transesophageal echo probe to the right from the midesophageal four-chamber view. It crosses the midline to lie on the left side of the vertebral column at about the eight or ninth thoracic vertebra. ${ }^{11}$ The descending aorta in such a case can be visualized when the probe is manipulated to view the IVC and hepatic vein in lower esophageal position for confirmation of IVC cannula placement (Fig. 10 and Video 10).

Another important clue obtained on examining the descending thoracic aorta is the presence of major aorta-pulmonary collaterals arteries (MAPCAs) (Fig. 11 and Video 11). The presence of continuous flow with systolic prominence, in a vessel arising from the 


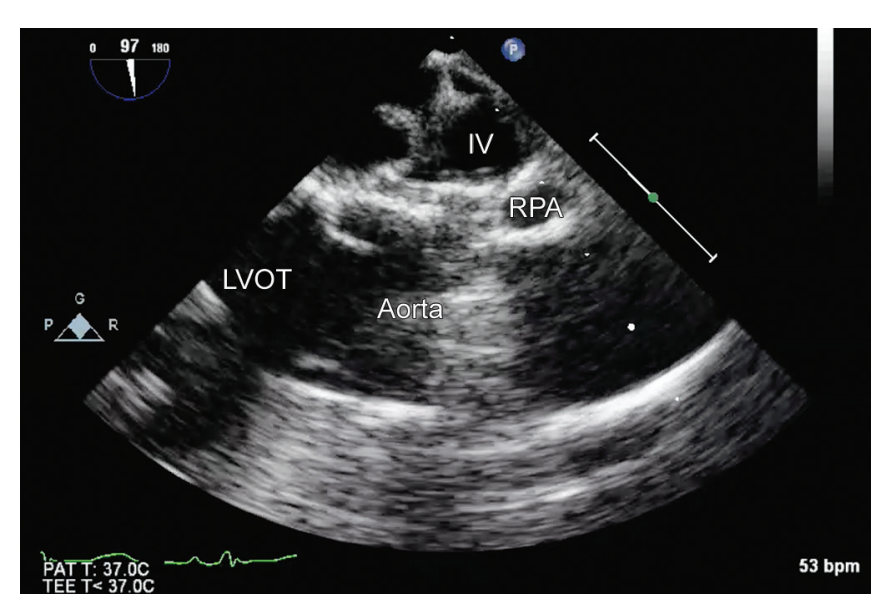

Fig. 8: Retroaortic innominate vein in a patient with tetralogy of Fallot. Note the innominate vein located behind the ascending aorta, adjacent to the right pulmonary artery. IV, Innominate vein; LVOT, Left ventricle outflow tract; RPA, Right pulmonary art

DTA, maybe a MAPCA. ${ }^{12}$ The location of such a vessel can be communicated to the surgeon who can confirm its presence and ligate it before going on $\mathrm{CPB}$, as was done in the depicted case. The left ventricle is also unlikely to be small and likely to be hyperdynamic in the presence of multiple MAPCAs.

At the other end of the spectrum is the TOF within the adequate size of the left ventricle (LV) presenting for surgery. The low pulmonary blood flow in TOF causes a low pulmonary venous return, which in turn results in a small LV by the flow-grow phenomenon. A too small LV can, however, fail after intracardiac repair because of the increased volume load. Although mitral annulus and LV length can be used to estimate the size of LV, $\mathrm{LV}$ end-diastolic volume is the preferred parameter for judging this. If LV end-diastolic volume is found to be less than $20 \mathrm{ml} / \mathrm{sqm}$, it may be preferable to undergo a staged approach, ${ }^{13}$ starting with a shunt, as was done in the case depicted in (Fig. 12 and Video 12).

TEE plays a significant role in the postoperative evaluation of the TOF patient in the evaluation of the VSD patch, RVOTO, pulmonary regurgitation, and myocardial function. While pressure recording of the left ventricle, right ventricle, and pulmonary arteries can alert us to the presence of residual RVOTO, a good preoperative image record forms the basis for further evaluation. As the initial obstruction in TOF can be anywhere from low infundibulum to beyond the main pulmonary arteries, TEE can locate the residual obstruction. An example is shown in (Fig. 13 and Video 13), where the residual obstruction was found to be just below the patch. This was communicated to the surgeon who later corrected it. A residual obstruction in TOF can potentially manifest as double chamber right ventricle later in life with features of right ventricle failure (Fig. 14 and Video 14).

On the corollary, the nature of a residual obstruction will also need to be determined. Evaluation of the nature of the obstruction and its variation with cardiac cycle by echocardiography is suggested to differentiate fixed and dynamic obstruction. Diffuse obstruction with an increase in dimension in diastole tilts the diagnosis in favor of dynamic obstruction (Fig. 15 and Video 15), while discrete obstruction with no change
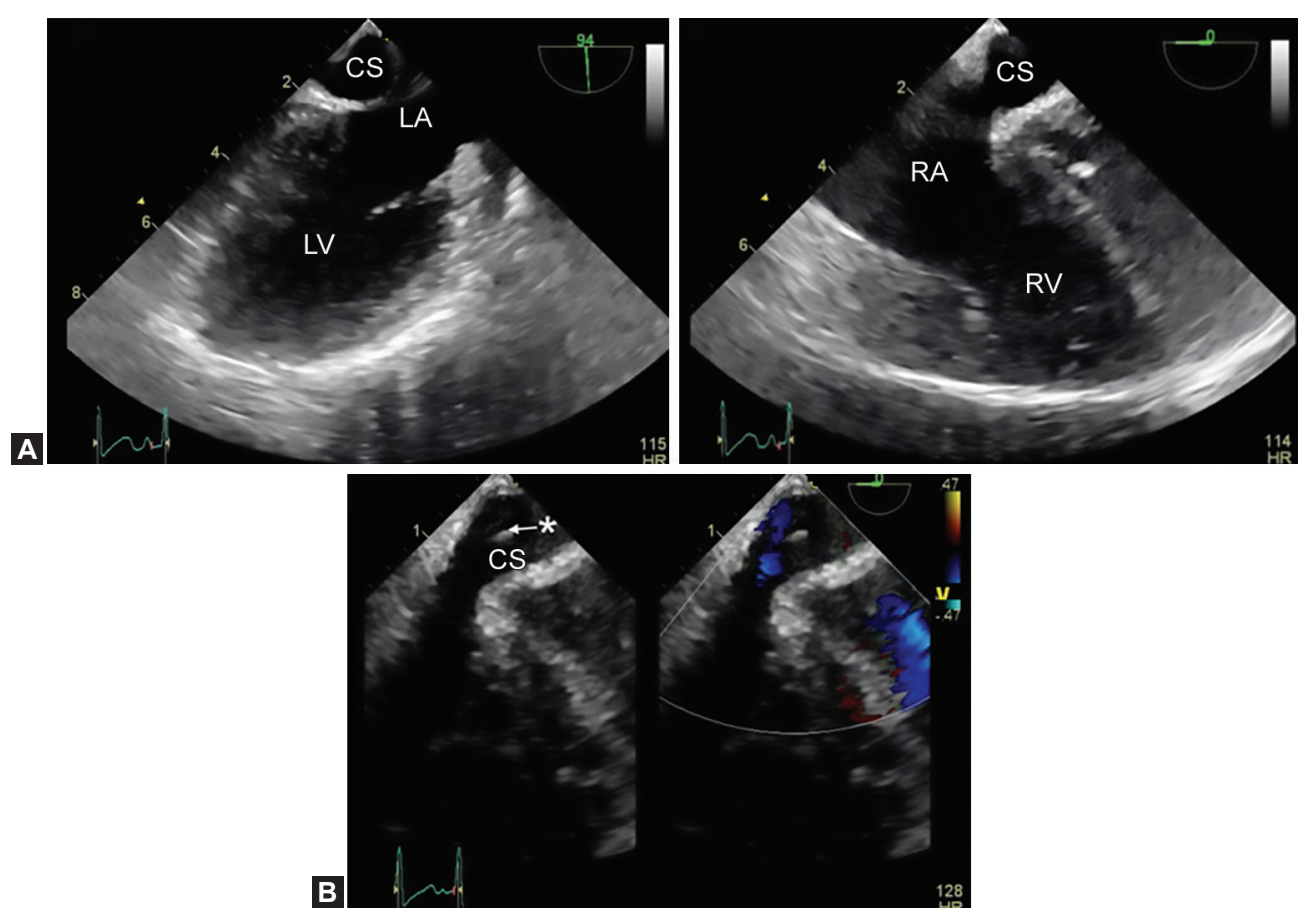

Fig. 9: Dilated coronary sinus in a tetralogy of Fallot patient who was detected to have a persistent left superior vena cava. (A) Midesophageal two chamber view, (B) Coronary sinus visualised by slightly advancing the probe from the midesophageal four chamber view 


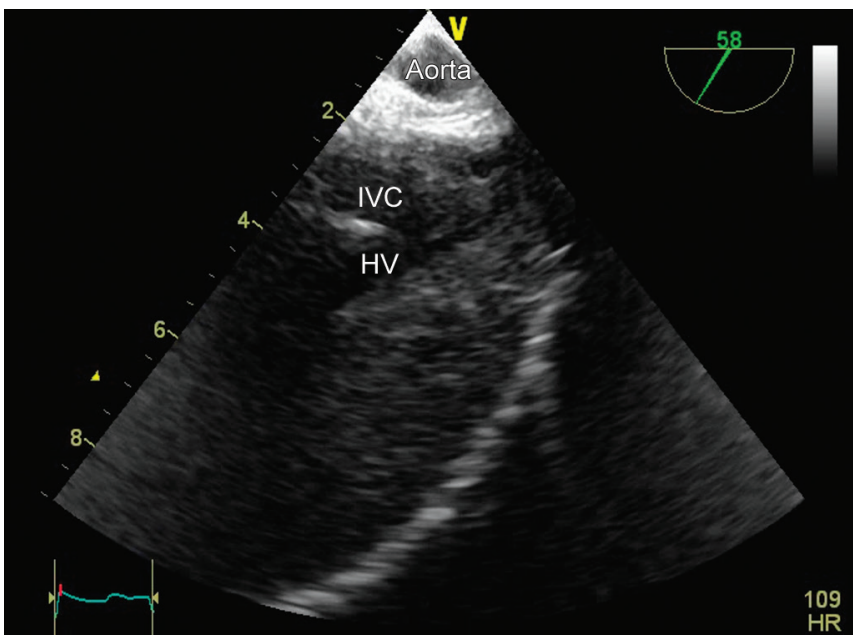

Fig. 10: Descending aorta located anterior to the esophagus in a tetralogy of Fallot patient with right sided aortic arch, with the probe placed in the lower esophageal position to visualize the inferior vena cava and hepatic vein. HV, Hepatic vein; IVC, Inferior vena cava

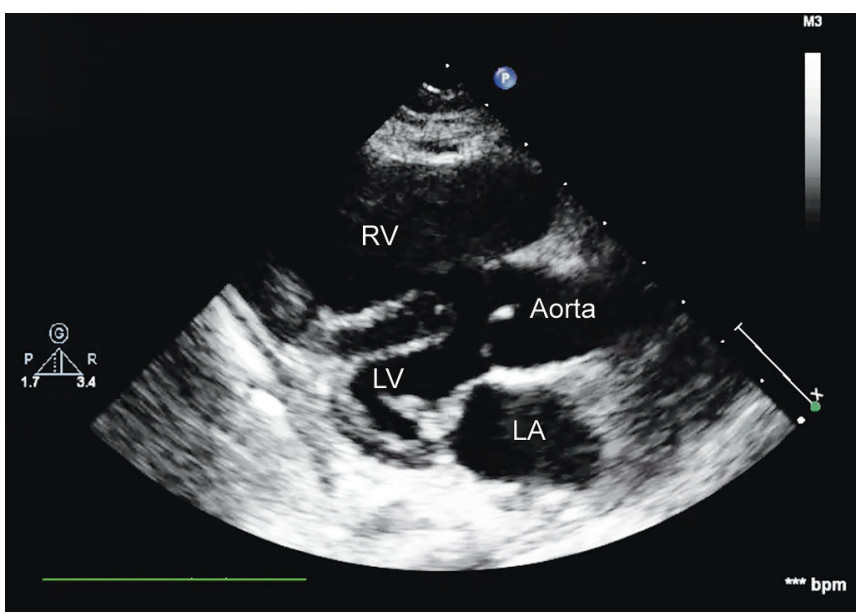

Fig. 12: Parasternal long axis view in a tetralogy of Fallot patient with a small left ventricle and posted for shunt procedure. LA, Left atrium; LV, Left ventricle; RV, Right ventricle.

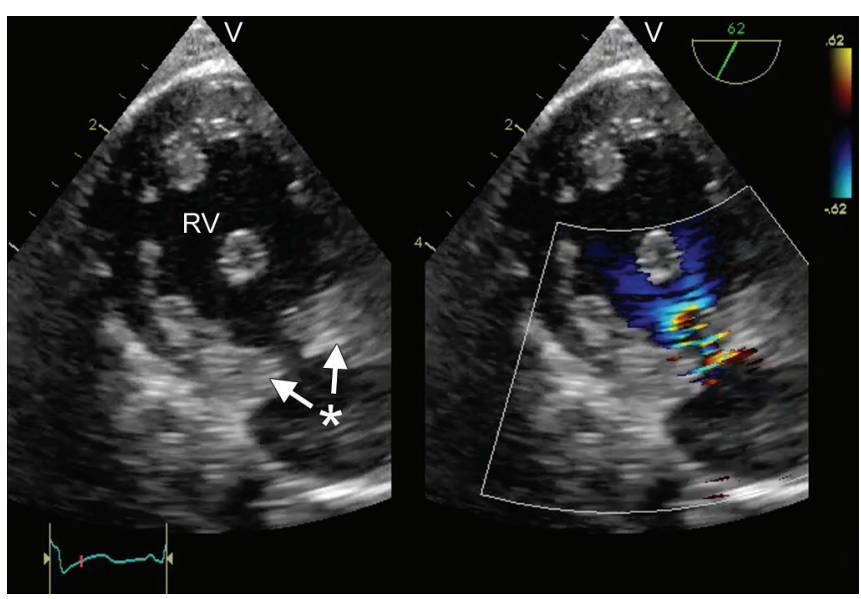

Fig. 14: Transgastric right ventricle outflow view showing double chamber right ventricle in a tetralogy of Fallot patient presenting after 1 year after repair. *, Muscle bundle in right ventricle outflow tract

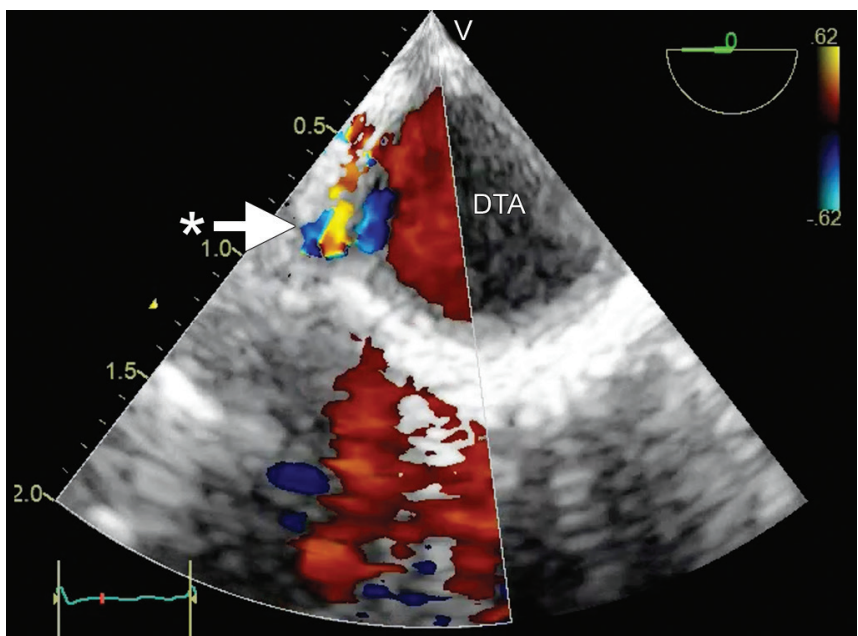

Fig. 11: Descending thoracic aorta giving rise to a vessel which was later confirmed to be a major aortopulmonary collateral artery. DTA, Descending thoracic aorta. *, Major aortopulmonary collateral artery

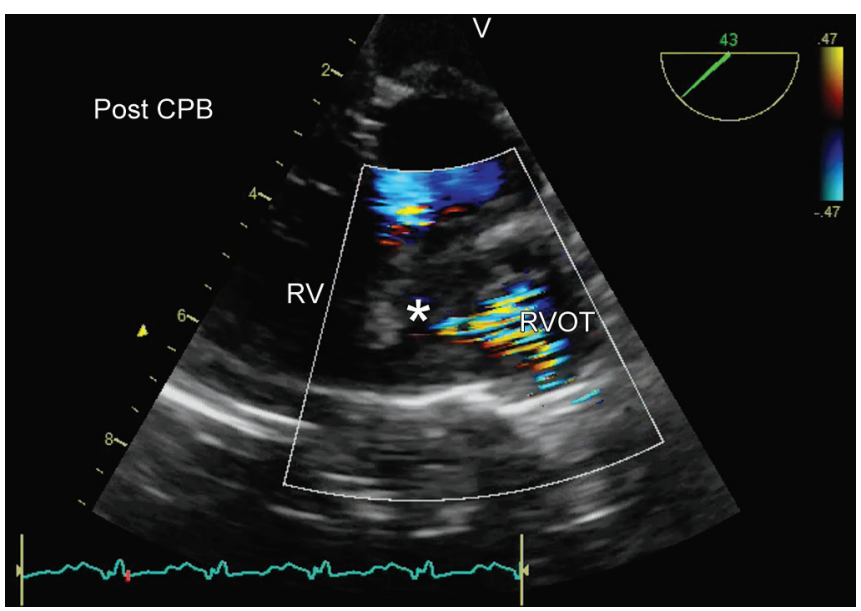

Fig. 13: Mid-esophgeal right ventricle inflow outflow view showing residual obstruction below the infundibular patch in a repaired tetralogy of fallot patient. RV, Right ventricle; RVOT, Right ventricle ouflow tract

in dimension during the cardiac cycle favors fixed obstruction, which needs correction. ${ }^{14}$ Apart from this, attention needs to be paid to the loading conditions when assessing the RVOT gradient and pressure ratio between the right ventricle and left ventricle. For example, in the case depicted in Figure 15, the initially high right ventricle outflow gradient reduced with the reduction in the abnormally high heart rate with the use of beta blockers.

The anesthetists' knowledge of these common variations in the TOF patient along with an efficient communication with the surgeon can serve to improve the patient outcome, not only in the peri-operative period but also in the long term. 

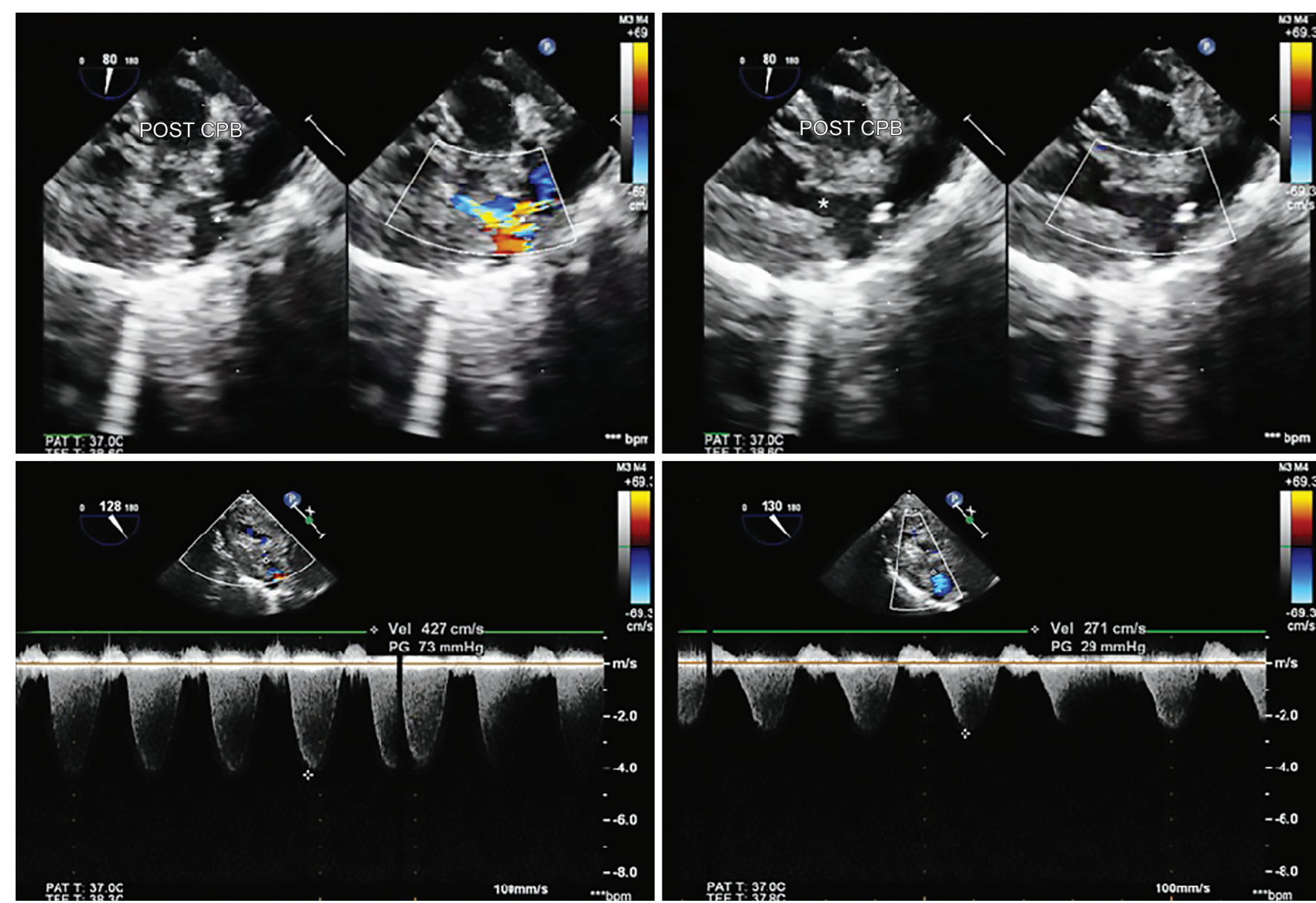

Fig. 15: Dynamic outflow obstruction persisting after surgical relief of right ventricle outflow obstruction in a neonate. (A) Systolic frame of midesophageal right ventricle outflow demonstrating diffuse hypertrophy and turbulence in right ventricle outflow.

\section{REFERENCES}

1. Puri GD, Raj R, Tacy TA.Transesophageal Echocardiography for Tetralogy of Fallot Repair: What a perioperative physician need to know? J Perioper Echocardiogr 2014;(2):51-57.

2. Quiñones M, Otto C, Stoddard M, Waggoner A, Zoghbi W. Recommendations for quantification of Doppler echocardiography: A report from the Doppler quantification task force of the nomenclature and standards committee of the American Society of Echocardiography. Journal of the American Society of Echocardiography. 2002;15(2):167-184.

3. Varghese R, Devendran V, Anjith P, Singhi A, Jesudian V, Sheriff E et al. Tetralogy of Fallot with subarterial ventricular septal defect: Surgical outcome in the current era. Annals of Pediatric Cardiology. 2015;8(1):4-9

4. Flanagan M, Foran R, Van Praagh R, Jonas R, Sanders S. Tetralogy of Fallot with obstruction of the ventricular septal defect: Spectrum of echocardiographic findings. Journal of the American College of Cardiology. 1988;11(2):386-395.

5. Sarris G. Tetralogy Surgery - Back To Baltimore 70 Years Later: Melbourne Heritage and Group Tribute to Juan Comas. Seminars in Thoracic and Cardiovascular Surgery: Pediatric Cardiac Surgery Annual. 2017;20:84-88.

6. Sukulal K, Bijulal S, Tharakan J. Differential diagnosis of vascular structures in relation to upper ascending aorta: The retro-aortic innominate vein. Annals of Pediatric Cardiology. 2013;6(1):97-98

7. Kulkarni S, Jain S, Kasar P, Garekar S, Joshi S. Retroaortic left innominate vein - Incidence, association with congenital heart defects, embryology, and clinical significance. Annals of Pediatric Cardiology. 2008;1(2):139-141

8. Chen S, Liu K, Chen H, Chiu I, Lee W, Wu Met al. Anomalous Brachiocephalic Vein: CT, Embryology, and Clinical Implications. American Journal of Roentgenology. 2005;184(4):12351240.

9. Balasundaram S, Al-Halees Z, Duran CG. Persistent left superior vena cava: a simple technique for adequate drainage during cardiopulmonary bypass. J Cardiovasc Surg 1991;32: 59-61.

10. Hastreiter A, D'Cruz I, Cantez T, Namin E, Licata R. Rightsided aorta. I. Occurrence of right aortic arch in various types of congenital heart disease. II. Right aortic arch, right descending aorta, and associated anomalies. Heart. 1966;28(6):722-739.

11. Edwards J. Anomalies of the Derivatives of the Aortic Arch System. Medical Clinics of North America. 1948;32(4): 925-949.

12. Aggarwal N, Sreedhar R, Gadhinglajkar S, Dharan B, Babu S, Pillai $\mathrm{M}$ et al. Intraoperative Diagnosis of Major Aortopulmonary Collateral Arteries by Transesophageal Echocardiography. Journal of Cardiothoracic and Vascular Anesthesia. 2018;32(4): 1794-1799.

13. Zhao K, Wang H, Han H, Sun J, Yin Z, Fang M et al. Staged Procedures versus Primary Repair for Tetralogy of Fallot and Small Left Ventricle. The Heart Surgery Forum. 2012;15(1):37.

14. Kaushal S, Radhakrishanan S, Dagar K, Iyer P, Girotra S, Shrivastava $\mathrm{S}$ et al. Significant intraoperative right ventricular outflow gradients after repair for tetralogy of Fallot: to revise or not to revise?. The Annals of Thoracic Surgery. 1999;68(5): 1705-1712. 